\title{
Keeping Denial-of-Service Attackers in the Dark*
}

\author{
Gal Badishi ${ }^{\dagger} \quad$ Amir Herzberg ${ }^{\ddagger} \quad$ Idit Keidar $^{\dagger}$
}

\begin{abstract}
We consider the problem of overcoming (Distributed) Denial of Service (DoS) attacks by realistic adversaries that have knowledge of their attack's successfulness, e.g., by observing service performance degradation, or by eavesdropping on messages or parts thereof. A solution for this problem in a high-speed network environment necessitates lightweight mechanisms for differentiating between valid traffic and the attacker's packets. The main challenge in presenting such a solution is to exploit existing packet filtering mechanisms in a way that allows fast processing of packets, but is complex enough so that the attacker cannot efficiently craft packets that pass the filters. We show a protocol that mitigates DoS attacks by adversaries that can eavesdrop and (with some delay) adapt their attacks accordingly. The protocol uses only available, efficient packet filtering mechanisms based mainly on (addresses and) port numbers. Our protocol avoids the use of fixed ports, and instead performs 'pseudo-random port hopping'. We model the underlying packet-filtering services and define measures for the capabilities of the adversary and for the success rate of the protocol. Using these, we provide a novel rigorous analysis of the impact of DoS on an end-to-end protocol, and show that our protocol provides effective DoS prevention for realistic attack and deployment scenarios.
\end{abstract}

Index Terms: Denial-of-Service, Port hopping, Secure two-party communication.

\footnotetext{
${ }^{*}$ A preliminary version of this paper appeared in the International Symposium on Distributed Computing (DISC) 2005.

${ }^{\dagger}$ Department of Electrical Engineering, The Technion - Israel Institute of Technology.

${ }_{\ddagger}^{\ddagger}$ Department of Computer Science, Bar Ilan University.
} 


\section{Introduction}

Denial of service (DoS) attacks have proliferated in recent years, causing severe service disruptions [6]. The most devastating attacks stem from distributed denial of service (DDoS), where an attacker utilizes multiple machines (often thousands) to generate excessive traffic [15]. Due to the acuteness of such attacks, various commercial solutions and off-the-shelf products addressing this problem have emerged. The main goal of all solutions is to provide lightweight packet-filtering mechanisms that are adequate for use in high-speed networks, where per-packet analysis must be efficient.

The most common solution uses an existing firewall/router (or protocol stack) to perform ratelimiting of traffic, and to filter messages according to header fields like address and port number. Such mechanisms are cheap and readily available, and are therefore very appealing. Nevertheless, rate-limiting indiscriminately discards messages, and it is easy to spoof (fake) headers that match the filtering criteria: an attacker can often generate spoofed packets containing correct source and destination IP addresses, and arbitrarily chosen values for almost all fields used for filtering ${ }^{1}$. Therefore, the only hope in using such efficient filtering mechanisms to overcome DoS attacks lies in choosing values that are unknown to the adversary. E.g., TCP's use of a random initial sequence number is a simple version of this approach, but is inadequate if the attacker has some (even limited) eavesdropping capability.

More effective DoS solutions are provided by expensive commercial devices that perform stateful filtering $[17,18,19]$. These solutions specialize in protecting a handful of commonly-used stateful protocols, e.g., TCP; they are less effective for stateless traffic such as UDP [19]. Such expensive solutions are not suitable for all organizations.

Finally, the most effective way to filter out offending traffic is using secure source authentication with message authentication codes (MAC), as in, e.g., IPSec [3]. However, this requires computing a MAC for every packet, which can induce significant overhead, and thus, this approach may be even more vulnerable to DoS attacks. Specifically, it is inadequate for use in high-speed networks with high volumes of traffic.

\footnotetext{
${ }^{1}$ An exception is the TTL field of IP packets, which is automatically decremented by each router. This is used by some filtering mechanisms, e.g. BGP routers that receive only packets with maximal TTL value (255) to ensure the packets were sent by a neighboring router, and the Hop Counter Filtering proposal.
} 
Our goal is to address DoS attacks on end hosts, e.g., in corporate networks, assuming the network leading to the hosts is functional. (A complementary solution protecting the end network can be deployed at the ISP.) In this paper, we focus on fortifying the basic building block of two-party communication. Specifically, we develop a DoS-resistant datagram protocol, similar to UDP or raw IP. Our protocol has promising properties, especially in overcoming realistic attack scenarios where attackers can discover some of the control information included in protocol packets, as described in [1]. We assume that a realistic adversary can detect whether its attack is successful or not, and adjust its behavior accordingly. However, this adjustment takes some time, as it involves gathering information from the system, processing it to decide on the proper adjustment, and then notifying all the attacking nodes (massive attacks employ many nodes). We believe that our ideas, with some practical adjustments, have the potential to find their way into future DoS protection systems. E.g., these ideas can be integrated into IPSec [3]. Our formal analysis proves the effectiveness of our ideas, and thus shows that their realization into a working system is highly beneficial.

The key to exploiting lightweight mechanisms that can filter high-speed traffic is using a dual-layer approach: On the one hand, we exploit cheap, simple, and readily-available measures at the network layer. On the other hand, we leverage these network mechanisms at the application layer. The latter allows for more complex algorithms as it has to deal with significantly fewer packets than the network layer, and may have closer interaction with the application. The higher layer dynamically changes the filtering criteria used by the underlying layer, e.g., by closing certain ports and opening others for communication. It is important to note that the use of dynamically changing ports instead of a single wellknown port does not increase the chance of a security breach, as a single application is listening on all open ports.

The main contribution of our work is in presenting a formal framework for understanding and analyzing the effects of proposed solutions to the DoS problem. The main challenges in attempting to formalize DoS-resistance for the first time are: coming up with appropriate models for the attacker and the environment, modeling the functionality that can be provided by underlying mechanisms such as firewalls, and defining meaningful metrics for evaluating suggested solutions. We capture the functionality of a simple 
network-level DoS-mitigation solution by introducing the abstraction of a port-based rationing channel. It is important to note that our use of ports just serves as an example. In fact, any field that appears on all packets can be used as the filtering criterion, and our analysis and suggested protocol apply to all such fields. For simplicity, we henceforth use the term 'port' to refer to any filtering criterion that can be dynamically changed by the application level. Our primary metric of an end-to-end communication protocol's resistance to DoS attacks is success rate, which is the worst-case expected portion of valid application messages that successfully reach their destination, under a defined adversary class.

Having defined our model and metrics, we proceed to give a generic analysis of the communication success rate over a port-based rationing channel in different attack scenarios. We distinguish between directed attacks, where the adversary knows the port used, and blind attacks, in which the adversary does not know the port. Not surprisingly, we show that directed attacks are extremely harmful: with as little as 100 machines (or a sending capacity 100 times that of the protocol) the success rate is virtually zero. On the other hand, the worst-case success rate that an attacker can cause in blind attacks in realistic scenarios is well over $90 \%$ even with 10,000 machines.

Our goal is therefore to "keep the attacker in the dark", so that it will have to resort to blind attacks. Our basic idea is to change the filtering criteria (i.e., ports) in a manner that cannot be predicted by the attacker. This port-hopping approach mimics the technique of frequency hopping spread spectrum in radio communication [20]. We assume that the communicating parties share a secret key unknown to the attacker; they apply a pseudo-random function [8] to this key in order to select the sequence of ports they will use. Note that such port-hopping has negligible effect on the communication overhead for realistic intervals between hops, and thus can be used even in high-speed networks. The remaining challenge is synchronizing the processes, so that the recipient opens the port currently used by the sender. We present a protocol for doing so in a realistic partially synchronous model, where processes are equipped with bounded-drift bounded-skew clocks, and message latency is bounded.

The paper proceeds as follows: Section 2 details related work. Section 3 details our models for the communication channel and the adversary. Section 4 provides generic DoS analysis. Section 5 describes our port-hopping protocol and analyzes its effectiveness. Section 6 concludes. 


\section{Related Work}

Our work continues the main line of research on prevention of Distributed Denial of Service attacks, which focuses on filtering mechanisms to block and discard the offending traffic. Our work is unique in providing rigorous model and analysis, which constitute the first step in formally modeling and evaluating the effectiveness of possible filtering and rate limiting mechanisms. Since our formal framework is not restricted to port-based filtering, but rather operates with any filtering based on per-packet fields, our model and analysis can be used in evaluating future protocols, and may assist in examining and comparing the solutions that exist now.

Most closely related is the work on SOS [12], followed by the work on Mayday [1]. Both propose realistic and efficient mechanisms that do not require global adoption, yet allow a server to provide services immune to DDoS attacks. These solutions, like ours, utilize efficient packet-filtering mechanisms between the server and predefined, trusted 'access point' hosts. The basic ideas of filtering based on ports or other simple identifiers ('keys'), and even of changing them, already appear in [1, 12], but without analysis and details. Additionally, [1] provides a discussion of attack types and limitations, justifying much of our model, including the assumption that the exposure of the identifier (port) number may be possible but not immediate. Furthermore, [1] mentions blind and targeted attacks (where blind attacks are attacks in which the adversary does not know the valid identifier), and asserts that the damage to the system is much more severe when targeted attacks are launched. We prove that this is indeed the case, and give exact quantities for the maximum performance degradation in both attack scenarios. Both SOS and Mayday require the setup of an overlay network consisting of several nodes, and effectively changes the prospective attack target to some nodes in the system (called SOAPs). In contrast, our solution does not require additional hosts, and is simple to construct and maintain.

There are other several proposed methods to filter offending DoS traffic. Some proposals, e.g., [10, 13], filter according to the source IP address. This is convenient and efficient, allowing implementation in existing packet filtering routers. However, IP addresses are subject to spoofing; furthermore, using a white-list of source addresses of legitimate clients/peers is difficult, since many hosts may have dynamic IP addresses due to the use of NAT, DHCP and mobile-IP. Some proposals try to detect spoofed senders, 
using new routing mechanisms such as 'path markers' supported by some or all of the routers en route, as in Pi, SIFF, AITF, and Pushback [21, 22, 2, 14], but global router modification is difficult to achieve. Few proposals try to detect spoofed senders using only existing mechanisms, such as the hop count (TTL), as in $\mathrm{HCF}$ [9]. However, empirical evaluation of these approaches show rather disappointing results [5].

A different approach is to perform application-specific filtering for pre-defined protocols [11, 16]. Such protection schemes are cumbersome, only work for a handful of well-known protocols, and are usually restricted to attackers that transmit invalid protocol packets.

In earlier work, we have presented Drum [4], a gossip-based multicast protocol resistant to DoS attacks. Drum does not make use of pseudo-random port-hopping, and it heavily relies on well-known ports that can be easily attacked. Therefore, Drum is far less resistant to DoS attacks than the protocol we present here. Finally, Drum focuses on multicast only, and as a gossip-based protocol, it relies on a high level of redundancy, whereas the protocol presented herein sends very little redundant information.

\section{Model and Definitions}

We consider a realistic semi-synchronous model, where processes have continuously-increasing local clocks with bounded drift $\Phi$ from real time. Each party may schedule events to occur when its local clock reaches a specific value (time). There is a bound $\Delta$ on the transmission delay, i.e., every packet sent either arrives within $\Delta$ time units, or is considered lost. Notice that while we assume messages always arrive within $\Delta$ time, this is only a simplification, and our results are valid even if a few messages arrive later than that; therefore, $\Delta$ should really be thought of as the typical maximal round trip time, and not as an absolute bound on a message's lifetime (e.g., a second rather than 60 seconds).

Our goal is to send messages from a sender $A$ to a recipient $B$, in spite of attempts to disrupt this communication by an adversary. The basic technique available to the adversary is to clog the recipient by sending many packets. The standard defense deployed by most corporations is to rate-limit and filter packets, typically by a firewall. We capture this type of defense mechanism using a port-based rationing channel machine, which models the communication channel between $A$ and $B$ as well as the filtering 
mechanism. To send a message, $A$ invokes a $c h \_s e n d(m)$ event, a message is received by the channel in a $n e t \_r e c v(m)$ event, and $B$ receives messages via $\operatorname{ch} \_r e c v(m)$ events. We assume that the adversary cannot clog the communication to the channel, and that there is no message loss other than in the channel. The channel discards messages when it performs rate-limiting and filtering.

The channel machine is formally defined in Appendix A. We now provide an intuitive description of its functionality. Since we assume that the attacker can spoof packets with valid addresses, we cannot use these addresses for filtering. Instead, the channel filters packets using port numbers, allowing deployment using existing, efficient filtering mechanisms. Specifically, let the set of port numbers be $\{1, \ldots, \psi\}$. The buffer space of the channel is a critical resource. The channel's interface includes the alloc action, which allows $B$ to break the total buffer space of $R$ messages into a separate allocation of $R_{i}$ messages per port $i \in\{1, \ldots, \psi\}$, as long as $R \geq \sum_{i=1}^{\psi} R_{i}$. For simplicity, we assume that the buffers are read and cleared together in a single deliver event, which occurs exactly once on every integer time unit. If the number of packets sent to port $i$ since the last deliver exceeds $R_{i}$, a uniformly distributed random subset of $R_{i}$ of them is delivered.

We define several parameters that constrain the adversary's strength. The most important parameter is the attack strength, $C$, which is the maximal number of messages that the adversary may inject to the channel between two deliver events.

As shown in [1], attackers can utilize different techniques to try to learn the ports numbers expected by the filters (and used in packets sent by the sender). However, these techniques usually require considerable communication and time. To simplify, we allow the adversary to eavesdrop by exposing messages, but we assume that the adversary can expose packets no earlier than $\mathcal{E}$ time after they are sent, where $\mathcal{E}$ is the exposure delay parameter. The exposure delay reflects the time it takes an attacker to expose the relevant information, as well as to distribute it to the (many) attacking nodes, possibly using very limited bandwidth (e.g., if sending from a firewalled network). Our protocol works well with as little as $\mathcal{E}>5 \Delta$.

Since the adversary may control some behavior of the parties, we take a conservative approach and let the adversary schedule the $a p p \_s e n d(m)$ events in which the application (at $A$ ) asks to send $m$ to $B$. To prevent the adversary from abusing these abilities by simply invoking too many app_send events before 
a deliver event, we define the throughput, $T \geq 1$, as the maximal number of app_send events in a single time unit. We further assume that $R \geq \Delta T$, i.e. that the capacity of the channel is sufficient to handle the maximal rate of app_send events.

Since we focus on connectionless communication such as UDP, our main metric for resiliency to DoS attacks is its success rate, namely the probability that a message sent by $A$ is received by $B$.

Definition 1 (Success rate $\mu$ ). Let $E$ be any execution of a given two-party protocol operating over a given port-based rationing channel with parameters $\Psi, R, C, \Phi, \Delta, \mathcal{E}$ and $T$, with adversary ADV. Let end $(E)$ be the time of the last deliver event in E. Let sent $(E)(r e c v(E))$ be the number of messages sent (resp., received) by the application, in app_send (resp., app_recv) events during E, prior to end $(E)-\Delta$

(resp, end $(E)$ ). The success rate $\mu$ of $E$ is defined as $\mu(E)=\frac{\operatorname{recv}(E)}{\operatorname{sent}(E)}$. The success rate of adversary $A D V$ is the average success rate over all executions of $A D V$. The success rate of the protocol, denoted $\mu(\Psi, R, C, \Phi, \Delta, \mathcal{E}, T)$, is the worst success rate over all adversaries $A D V$.

Finally, a protocol can increase its success rate by sending redundant information, e.g., multiple copies or error-correcting codes. We therefore also consider a system's message (bit) complexity, which is the number of messages (resp. redundant bits) sent on the channel per each application message.

\section{Analyzing the Success Rate in a Single Slot with a Single Port}

This section provides generic analysis of the probability of successfully communicating over a port-based rationing channel under different attacks, when messages are sent to a single open port, $p$. This analysis is independent of the timing model and the particular protocol using the channel, and can therefore serve to analyze different protocols that use such channels, e.g., the one we present in the ensuing section. We focus on a single deliver event, and analyze the channel's delivery probability, which is the probability for a valid message in the channel's buffer to be delivered, in that event. Since every $c h \_s e n d(m)$ event eventually results in $m$ being added to the channel's buffer, we can use the channel's delivery probability to analyze the success rates of higher level protocols.

Let $R_{p}$ denote the ration allocated to port $p$ in the last alloc event, and let $\operatorname{In}(p)$ be the contents of 
the channel's buffer for port $p$ (see Appendix A for more details). Consider a deliver event of a channel from $A$ to $B$, when $A$ sends messages only to port $p$. We introduce some notations:

$R_{p}=R$ is the value of the channel's $R_{p}$ when deliver occurs.

$a_{p}=a$ is the number of messages whose source is $A$ in the channel's $\operatorname{In}(p)$ when deliver occurs. We assume $a \leq R$. If $a_{p}<R_{p}$ (i.e., $a<R$ ), we say that there is over-provisioning on port $p$.

$c_{p}$ is the number of messages whose source is not $A$ in $\operatorname{In}(p)$ when deliver occurs.

Assume that $1 \leq a \leq R$. If $c_{p}<R-a+1$ then $B$ receives $A$ 's messages, and the attack does not affect the communication from $A$ to $B$ on port $p$. Let us now examine what happens when $c_{p} \geq R-a+1$.

Lemma 1. If $c_{p} \geq R-a+1$, then the channel's delivery probability is $\frac{R}{c_{p}+a}$.

Proof. The channel delivers $m \in \operatorname{In}(p)$ if it is part of the $R$ messages read uniformly at random from the $c_{p}+a$ available messages. Thus, the delivery probability is $\frac{R}{c_{p}+a}$.

If the attacker knows that $B$ has opened port $p$, it can direct all of its power to that port, i.e., $c_{p}=C$, where we assume $C \geq R-a+1$. We call this a directed attack.

Corollary 1. In a directed attack at rate $C$ on B's port p, the delivery probability on the attacked port is $\frac{R}{C+a}$, assuming $1 \leq a \leq R$ and $C \geq R-a+1$.

Lemma 2. For fixed $R$ and $c_{p}$ such that $1 \leq a \leq R$ and $c_{p} \geq R-a+1$, the probability of $B$ receiving only invalid messages on port p decreases as a increases.

The proof of this lemma is simple and is omitted due to space considerations.

\subsection{Blind Attack}

We define a blind attack as a scenario where $A$ sends messages to a single open port, $p$, and the adversary cannot distinguish this port from a random one. We now analyze the worst-case delivery probability under a blind attack. 
In general, an adversary's strategy is composed both of timing decisions and injected messages. The timing decisions affect $a$, the number of messages from $A$ that are in the channel at a given delivery slot. Given that $a$ is already decided, we define the set of all strategies of an attacker with sending rate $C$ as:

$$
S(C) \triangleq\left\{\left\{c_{i}\right\}_{i \in \psi} \mid \forall i \in \psi: c_{i} \in \mathbb{N} \cup\{0\} \wedge \sum_{i=1}^{\psi} c_{i}=C\right\}
$$

Each strategy $s \in S$ is composed of the number of messages the attacker sends to each port. Note that since the adversary wishes to minimize the delivery probability, we restrict the discussion to the set of attacks that fully utilize the attacker's capacity for sending messages. We denote by $\mu_{B}(a, C, R)$ : $S(C) \rightarrow[0,1]$ the channel's delivery probability under all possible blind attack strategies with the given $a, C$, and $R$. Since $S$ is a finite set, $\mu_{B}$ has at least one minimum point, and we define the delivery probability to be that minimum:

$$
\mu_{B}(a, C, R) \triangleq \min _{s \in S(C)} \mu_{B}(a, C, R, s)
$$

We sometimes use $\mu_{B}$ instead of $\mu_{B}(a, C, R)$ when $a, C$, and $R$ are clear from context. We want to find lower bounds on $\mu_{B}$, depending on the attacker's strength. We say that port $p_{i}$ is attacked in strategy $s$ if $c_{p_{i}}>0$. We partition $S(C)$ according to the number of ports being attacked, as follows:

$$
S_{k} \triangleq\{s \in S(C) \mid \text { Exactly } k \text { ports are being attacked in } s\}
$$

In Appendix B we find a lower bound on $\mu_{B}$ as follows: We first derive a lower bound on $\left\{\mu_{B}\left(a, C, R, s_{k}\right) \mid s_{k} \in\right.$ $\left.S_{k}\right\}$; this lower bound is given as a function of $k$ in Corollary 2. Incidently, the worst degradation occurs when the attacker divides its power equally among the attacked ports, i.e., when it sends $\frac{C}{k}$ messages to each attacked port (this is proven in Lemma 5). Then, we show lower bounds on $\mu_{B}(a, C, R)$ by finding the $k$ that yields the minimum value. Finally, we prove the following lemma: 
Lemma 3. $\mu_{B}(a, C, R)$ is bounded from below by the following function $f(a, C, R)$ :

$$
f(a, C, R)= \begin{cases}\frac{\psi a}{C+\psi a} & \text { if } R=a \text { and } C \geq \psi \\ 1-\frac{C}{\psi(1+a)} & \text { if } R=a \text { and } C<\psi \\ \frac{\psi R}{C+\psi a} & \text { if } R>a \text { and } C \geq \frac{\psi a}{\sqrt{\frac{R}{R-a}}-1} \\ \frac{\psi a-C\left(\sqrt{\frac{R}{R-a}}-1\right)}{\psi a}+\frac{R}{\psi} \cdot \frac{C\left(\sqrt{\frac{R}{R-a}}-1\right)^{2}}{a^{2} \sqrt{\frac{R}{R-a}}} & \text { if } R>a \text { and } C<\frac{\psi a}{\sqrt{\frac{R}{R-a}}-1} \\ 0 & \text { otherwise }\end{cases}
$$

Lemma 3 provides us with some insights of the adversary's best strategy and of the expected degradation in delivery probability. If no over-provisioning is used (i.e., $R=a$ ), then the adversary's best strategy is to attack as many ports as possible. This is due to the fact that even a single bogus message to the correct port degrades the expected delivery probability. When the adversary has enough power to target all of the available ports with at least one message, it can attack with more messages per attacked port, and the delivery probability asymptotically degrades much like the function $\frac{1}{C}$. When not all ports are attacked, the adversary would like to use its remaining resources to attack more ports rather than target a strict subset of the ports with more than one bogus message per port. The degradation of the expected delivery probability is then linear as the attacker's strength increases.

When over-provisioning is used $(R>a)$, it affects the attack and its result in two ways. First, the attacker's best strategy may not be to attack as many ports as it can, since a single bogus message per port does not do any harm now. Second, for an adversary with a given strength, the degradation in delivery probability is lower when over-provisioning is used than when it is not employed. We can see in Equation 1 that if the attacker has enough power to attack all the ports, the over-provisioning ratio $\frac{R}{a}$ is also the ratio by which the delivery probability is increased, compared to the case where $R=a$. 


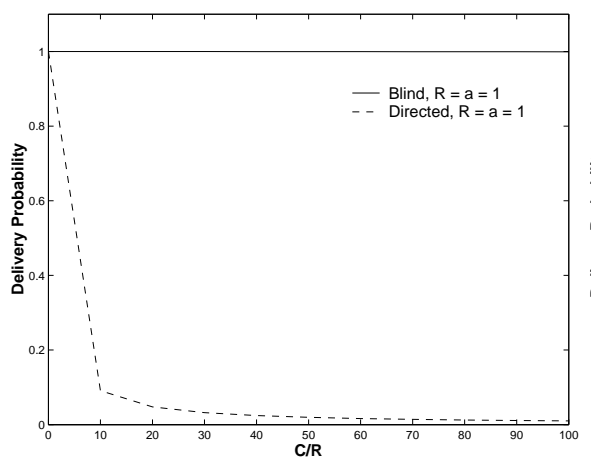

(a) Blind vs. Directed, $R=a=1$.

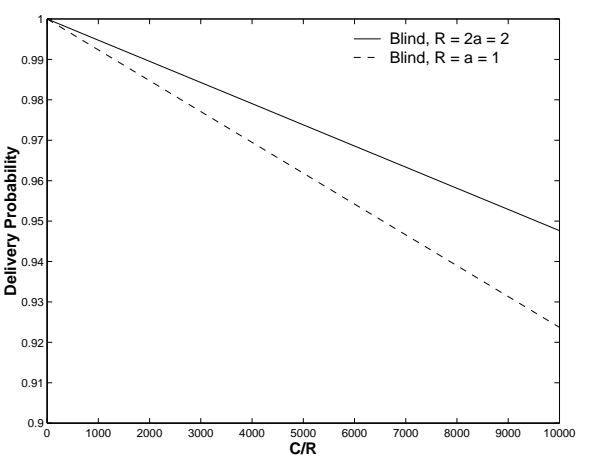

(b) Blind, $a=1$.

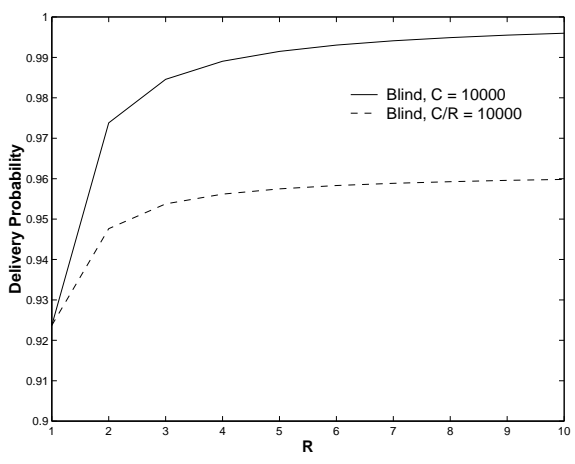

(c) Blind, $a=1$.

Figure 1: Delivery probability per slot in various attack scenarios on a single port, $\psi=65536$.

\subsection{Actual Values}

Figure 1 shows the expected worst-case delivery probabilities for various attack scenarios on a single port. For directed attacks, we show the actual delivery probability, and for blind attacks, the lower bound $f(a, C, R)$ is shown. We chose $\psi=65536$, the number of ports in common Internet protocols, e.g., UDP. Figure 1(a) illustrates the major difference between a directed attack and a blind one: even for a relatively weak attacker $(C \leq 100)$, the delivery probability under a directed attack approaches 0 , whereas under a blind attack, it virtually remains 1 .

Figure 1(b) examines blind attacks by much stronger adversaries (with $C$ up to 10,000 for $R=1$, and up to 20,000 for $R=2$ ). We see that the delivery probability gradually degrades down to a low of $92.5 \%$ when $R=1$. If we use an over-provisioned channel, i.e., have $a=1$ (one message from $A$ ) when $R=2$, the delivery probability improves to almost $95 \%$ for $C=20,000$. (The ratio $\frac{C}{R}$ is the same for both curves). Figure 1(c) shows the effect of larger over-provisioning. We see that the cost-effectiveness of over-provisioning diminishes as $\frac{R}{a}$ increases.

\section{DoS-Resistant Communication}

We now describe a protocol that allows for DoS-resistant communication in a partially-synchronous environment. The protocol's main component is an ack-based protocol. $B$ sends acknowledgments (acks) for messages it receives from $A$, and these acks allow the parties to hop through ports together. 
However, although the ack-based protocol works well as long as the adversary fails to attack the correct port, once the adversary gets a hold of the port used, it can perform a directed attack that renders the protocol useless. By attacking the found data port, or simultaneously attacking the found data and ack ports, the adversary can effectively drop the success rate to 0 , and no port hopping will occur. To solve this matter, there is a time-based proactive reinitialization of the ports used for the ack-based protocol, independent of any messages passed in the system.

\subsection{Ack-Based Port Hopping}

We present an ack-based port-hopping protocol, which uses two port-based rationing channels, from $B$ to $A$ (with ration $R_{B A}$ ) and vice versa (with ration $R_{A B}$ ). For simplicity we assume $R_{A B}=2 R_{B A}=2 R$. $B$ always keeps two open ports for data reception from $A$, and $A$ keeps one port open for acks from $B$. The protocol hops ports upon a successful round-trip on the most recent port used, using a pseudorandom function, $P R F^{* 2}$. In order to avoid hopping upon adversary messages, all protocol messages carry authentication information, using a second pseudo-random function, $P R F$, on $\{0,1\}^{\kappa}$. (We assume that $P R F$ and $P R F^{*}$ use different parts of $A$ and $B$ 's shared secret key.)

The protocol's pseudocode appears in Figure 2. Both $A$ and $B$ hold a port counter $P$, initialized to some seed (e.g., 1). Each party uses its counter $P$ in order to determine which ports should be open, and which ports to send messages to. $B$ opens port $p_{\text {old }}$ using the $(P-1)^{\text {th }}$ element in the pseudo-random sequence, and $p_{\text {new }}$, using $P$. A sends data messages to the $P^{\text {th }}$ port in the sequence, and opens the $P^{t h}$ port in a second pseudo-random sequence designated for acks. When $B$ receives a valid data message from $A$ on port $p_{\text {old }}$, it sends an ack to the old ack port. When it receives a valid message on port $p_{\text {new }}$, it sends an ack to the $P^{t h}$ ack port, and then increases $P$. When $A$ receives a valid ack on port $p_{a c k}$, it increases $P$. We note that several data messages may be in transit before a port hop takes place, since it takes at least one round-trip time for a port hop to take effect, and in a high-speed network, multiple

\footnotetext{
${ }^{2}$ Intuitively, we say that $f_{k e y}($ data $)$ is pseudo-random function $\left(P R F^{*}\right)$ if for inputs of sufficient length, it cannot be distinguished efficiently from a truly random function $r$ over the same domain and range, by a PPT adversary which can receive $g(x)$ for any values of $x$, where $g=r$ with probability half and $g=f$ with probability half. For definition and construction, see [8].
} 
PROTOCOL FOR SENDER $A$ :

$$
\begin{aligned}
& \text { On } a c k \_i n i t(s e e d): \quad \text { On } a p p \_s e n d(d a t a): \quad \text { On } c h \_r e c v\left(a c k, p_{a c k}\right) \text { : } \\
& P=\text { seed } \\
& m=\operatorname{data} \mid P R F_{S_{A B}}(P \mid \text { "data") } \\
& p_{a c k}=P R F_{S_{A B}}^{*}(P \mid \text { "ack" }) \quad c h \_s e n d\left(m, P R F_{S_{A B}}^{*}(P \mid \text { "data" })\right) \\
& \text { if ack.auth }=P R F_{S_{A B}}(P \mid \text { "ack" }) \text { then } \\
& \operatorname{alloc}\left(p_{\text {ack }}, 0\right) \\
& p_{a c k}=P R F_{S_{A B}}^{*}(P+1 \mid \text { "ack") } \\
& \text { alloc }\left(p_{\text {ack }}, R_{B A}\right) \\
& P=P+1
\end{aligned}
$$

PROTOCOL FOR RECIPIENT $B$ :

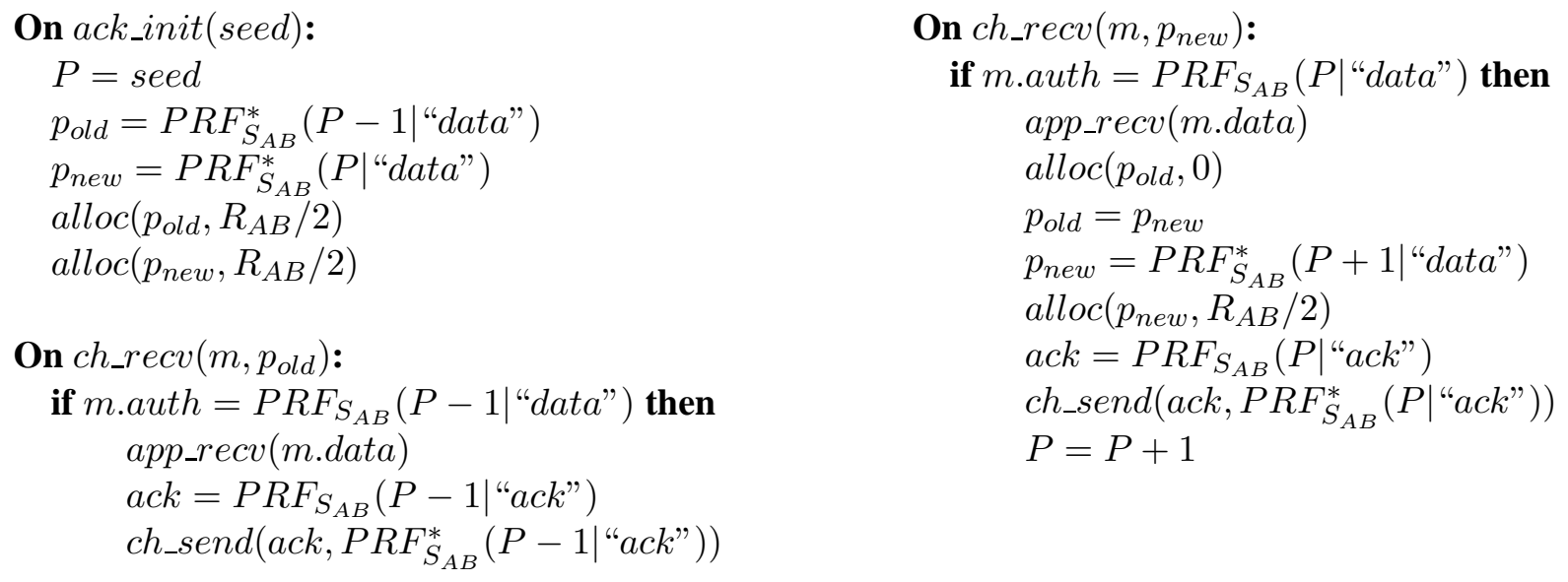

Figure 2: Two-party ack-based port-hopping.

messages are sent within this time span. The proof of the next theorem is given in Appendix C.

Theorem 1. When using the ack-based protocol, the probability that a data message that A sends to port p arrives when $p$ is open is 1 up to a polynomially-negligible factor ${ }^{3}$.

In order to compute the throughput that the protocol can support in the absence of a DoS attack (i.e., when $C=0$ ), we need to take latency variations into consideration. Since messages sent up to $\Delta$ time apart can arrive in the same delivery slot, a throughput $T \leq R / \Delta$ ensures $a \leq R$. For the rest of this section, we assume $T \leq R / \Delta$.

We now analyze the protocol's success rate under DoS attacks. We say that the adversary is in blind mode if it does not know the ports used by the protocol. We first give a lower bound on the success rate in blind mode, and then give a lower bound on the probability to be in blind mode at a given time $t$. Finally, $\mu$ is bounded by the probability to be in blind mode throughout the execution of the protocol, times the

\footnotetext{
${ }^{3}$ Namely, for every polynomial $g>0$, there is some $\kappa_{g}$ s.t. when $\kappa \geq \kappa_{g}$, then the success rate $\mu(t) \geq f(R, C, R)-g(\kappa)$.
} 
success rate in blind mode.

Suppose $B$ opens port $p$ with reception rate $R_{p}$, and that $a \leq R_{p}$ messages from $A$ are waiting in its channel, along with $c_{p}$ messages from the adversary $\left(c_{p} \geq 0\right)$. By Lemma 1 , the success rate monotonically non-increases with $a$. Since the adversary can control $a$ by varying the network delays, it can set $a$ as high as possible for a delivery slot. Therefore, the worst case occurs when $a=T \Delta$. Using Equation 1, we get that the success rate in blind mode is bounded from below by $f(T \Delta, C, R)$.

Note that the protocol begins in blind mode. We now analyze the probability that the protocol keeps the adversary in blind mode. The only way the adversary can learn of a port used by the protocol is using an expose event $\mathcal{E}$ time after a message is sent to that port. This information is only useful for an attack if the port is still in use. Let us trace the periodic sequence of events that causes the data port to change (once it changes, acks for the old port are useless). Assume $A$ continuously sends messages $m_{1}, m_{2}, \ldots$ to $B$ starting at time 0 , and consider an execution without an attack: (1) By time $\Delta, B$ receives a valid message from the channel and sends an ack to $A$; (2) By time $2 \Delta, A$ receives the ack and changes the sending port; (3) $B$ gets the last message destined for the old port at most at time $3 \Delta$.

If $\mathcal{E} \geq 3 \Delta$, the adversary remains in blind mode. Now let us examine what happens under attack. In order to prevent the port from changing, the adversary must either prevent $B$ from getting valid data messages or prevent $A$ from receiving acks. By Lemma 2, the probability that all valid messages are dropped decreases when $a$ increases. Thus, (as opposed to the previous analysis), in order to increase the probability that all valid messages are dropped, the adversary should set $a \leq 1$ whenever possible. Denote $\mu_{B}=f(1, C, R)$, the lower bound on the probability of a single message to be received on a single port given in Section 4.1.

Lemma 4. If $\mathcal{E}=2 k \Delta$ for $k>0$, and $A$ sends messages to $B$ at least every $2 \Delta$ time units, then the probability that the port changes while the attacker is still blind is at least $1-\left(1-\mu_{B}^{2}\right)^{k}$.

Proof. The probability that the port does not change in a single round-trip is at most $1-\mu_{B}^{2}$. Since $A$ sends messages to $B$ every $2 \Delta$ time units, at the conclusion of each maximal time round-trip, there is at least one new message on its own round-trip. In order for the port not to change while the adversary is still blind, every round-trip needs to fail. Since the attacker can react only after $2 k \Delta$ time, there is time 
for $k$ round-trips in which the attacker is blind, even if none of them succeed. The probability that all of them fail is less than $\left(1-\mu_{B}^{2}\right)^{k}$. If one succeeds, the port changes. And so, the probability that the port changes is at least $1-\left(1-\mu_{B}^{2}\right)^{k}$.

The lower bound above is illustrated in Figure 3(a).

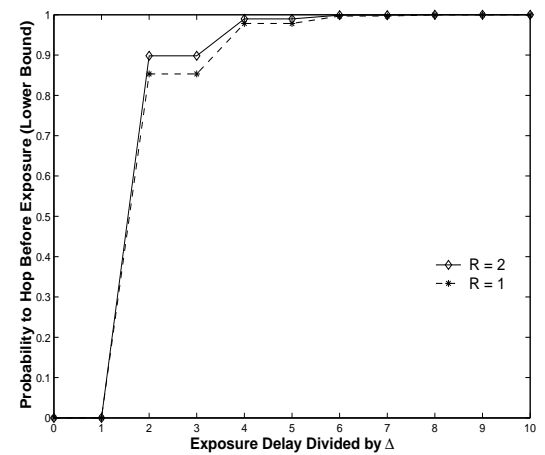

$\begin{array}{cccc}\text { (a) Bound on } & \text { prob. to } & \text { (b) } \\ \text { hop } & \text { before } & \text { exposure, to }\end{array}$ $\frac{C}{R}=10,000$

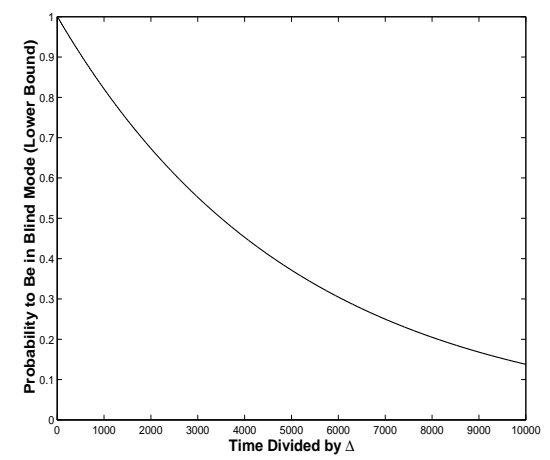

(b) Bound $(g)$ on prob. stay in blind mode, $R=1, C=10,000, \mathcal{E}=4 \Delta$

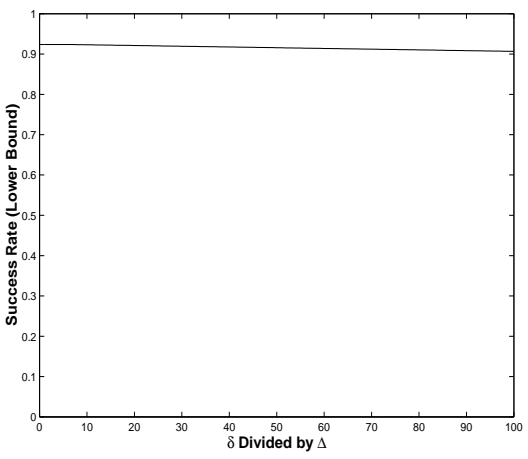

(c) Bound on protocol's success rate, $\Delta=T=R=1, C=10,000, \mathcal{E}=5 \Delta$

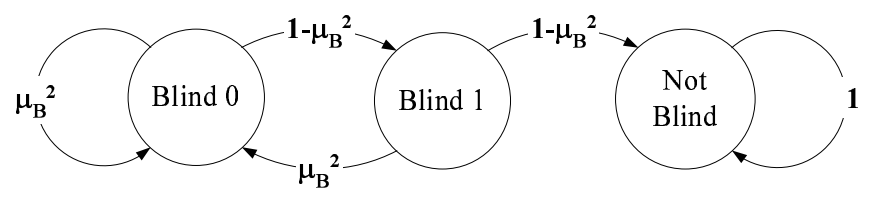

(d) Markov chain for computing the lower bound in Figure 3(b).

Figure 3: The effect of $\mathcal{E}$ on the ack-based protocol, $\psi=65536$.

We now bound the probability to be in blind mode at time $t$, by assuming that once the attacker leaves the blind mode it never returns to it. The bound is computed using a Markov chain, where each state is the number of round-trips that have failed since the last port change. In the last state, all round-trips have failed before the exposure, and thus the attacker is no longer blind. The Markov chain for $\mathcal{E}=4 \Delta$ is shown in Figure 3(d). We use the chain's transition matrix to compute the probability $g(t, \mathcal{E}, C, R)$ for remaining in blind mode at time $t$. Figure 3(b) shows values of $g$ for $\mathcal{E}=4 \Delta$. We can see that the protocol works well only for a limited time.

Finally, we note that the protocol's message complexity is 2 , since it sends an ack for each message, and its bit complexity is constant: $\log _{2}(\psi)$ bits for the port plus $\kappa$ bits for the authentication code. 


\subsection{Adding Proactive Reinitializations}

We now introduce a proactive reinitialization mechanism that allows choosing new seeds for the ackbased protocol depending on time and not on the messages passed in the system. We denote by $t_{A}(t)$ and $t_{B}(t)$ the local clocks of $A$ and $B$, resp., where $t$ is the real time. From Section 3 we get that $0 \leq\left|t_{A}(t)-t\right| \leq \Phi, 0 \leq\left|t_{B}(t)-t\right| \leq \Phi$. We also assume $t_{A}, t_{B} \geq 0$.

If $A$ reinitializes the ack-based protocol and then sends a message to $B$ at time $t_{A}\left(t_{0}\right)$, this message can reach $B$ anywhere in the real time interval $\left(t_{0}, t_{0}+\Delta\right]$. Therefore, the port used by $A$ at $t_{A}\left(t_{0}\right)$ must be open by $B$ at least throughout this interval. To handle the extreme case where $A$ sends a message at the moment of reinitialization, $B$ must use the appropriate port starting at time $t_{B}\left(t_{0}\right)-\Phi$. (We note that $t_{0}$ may also be $\Phi$ time units apart from $t_{A}\left(t_{0}\right)$.) We define $\delta$ as the number of time units between reinitializations of the protocol, and assume for simplicity and effectiveness of resource consumption that $\delta>4 \Phi+\Delta$ (see Figure 4 for more details).

Every $\delta$ time units, $A$ feeds a new seed to the ack-based protocol, and $B$ anticipates it by creating a new instance of the protocol, which waits on the new expected ports. Once communication is established using the new protocol instance, or once it is clear that the old instance is not going to be used anymore, the old instance is terminated. The pseudocode for the proactive reinitialization mechanism can be found in Figure 4. Due to space considerations we do not detail the change in port rations at the recipient's side as protocol instances are created or terminated. We also note that there is a negligible probability that more than one ack-based protocol instance will share the same port. Even if this happens, differentiating between instances can be easily done by adding the instance number (i.e., the total number of times a reinitialization was performed) to each message. The proof of the next theorem is given in Appendix D.

Theorem 2. When using the ack-based protocol with proactive reinitializations, the probability that a data message that A sends to port $p$ arrives when $p$ is open is 1 up to a polynomially-negligible factor.

Proactive reinitialization every $\delta$ time units allows us to limit the expected degradation in success rate for a single ack-based protocol instance. Choosing $\delta$ is therefore an important part of the combined protocol. A small $\delta$ allows us to maintain high success rate in the ack-based protocol, but increases the 
PROTOCOL ADD-ON FOR SENDER $A$ :

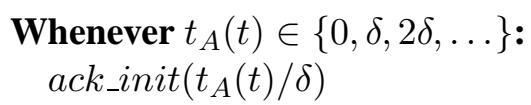

PROTOCOL ADD-ON FOR RECIPIENT $B$ :

When $t_{B}(t)=0$ :

Create the first ack-based protocol instance

For that instance, ack_init(0)
PROTOCOL ADD-ON FOR RECIPIENT $B$ (CONTINUED):

Whenever $\left(t_{B}(t)+2 \Phi\right) \in\{\delta, 2 \delta, 3 \delta, \ldots\}$ :

Create a new ack-based protocol instance

For that instance, ack_init $\left(\left(t_{B}(t)+2 \Phi\right) / \delta\right)$

$4 \Phi+\Delta$ time after creating a new ack-based protocol instance

or $\Delta$ time after receiving the first msg for this new instance:

Terminate all older protocol instances

Figure 4: Proactive reinitialization of the ack-based protocol.

average number of ports that are open in every time unit (due to running several protocol instances in parallel). When several ports are used the ration for each one of them is decreased, and so might the success rate. On the other hand, choosing a high $\delta$ entails lower success rate between reinitializations. We conclude the discussion above and the results presented in Section 5.1 with the following theorem:

Theorem 3. The success rate of the proactively reinitialized ack-based protocol with throughput $T \leq$ $R / \Delta$ and reinitialization periods of length $\delta$ is bounded from below by: $g(\delta+\Delta, \mathcal{E}, C, R) \cdot f(T \Delta, C, R)$ up to a polynomially-negligible factor.

Figure 3(c) shows the value of $g(\delta+1, \mathcal{E}, 10000,1) \cdot f(1,10000,1,1)$. We can see that the proactively reinitialized protocol's success rate stays over $90 \%$ even for $\delta=100 \Delta$, i.e., even for relatively long periods between reinitializations.

\section{Conclusions and Directions for Future Work}

We have presented a model for port-based rationing channels, and a protocol robust to DoS attacks, for communication over such channels. Our protocol is simple and efficient, and hence can sustain high loads of traffic, as happens, e.g., in high-speed networks. At the same time, our analysis shows that the protocol is highly effective in mitigating the effects of DoS attacks. Our formal framework and suggested protocol apply not only to port-based filtering, but to a much broader category of filtering based on any packet identifier. Thus, our work constitutes the first step in evaluating existing filtering and rate-limiting mechanisms. 
As the important field of application-level DoS mitigation is relatively new, there is much research space to explore. While our worst case analysis is valuable, it can be followed by simulations, experiments, and common case analysis. Moreover, the system aspects of deploying such a protocol in today's Internet are yet to be dealt with. We now describe several exemplary future research directions.

Our model is realistic, as it only requires the underlying channel to provide port-based filtering; therefore, it can be efficiently implemented using existing mechanisms, typically at a gateway firewall or router. This raises an interesting question regarding the trade-off between the cost and the possible added value of implementing additional functionality by the channel (e.g., at the firewall). We hope that future work will take further strides towards defining realistic yet tractable models of the channel and the adversary that will aid in answering this question.

This work has focused on two parties only. It would be interesting to extend it to multiparty scenarios, such as client-sever and multicast. These scenarios may require a somewhat different approach, and will obviously necessitate analyses of their own. Furthermore, we required the parties to share a secret key; we believe we can extend the solution to establish this key using additional parties, e.g., a key distribution center, or using 'proof of work' [7].

Our work has focused on resisting DoS attacks; however, it could impact the performance and reliability properties of the connection; in fact, it is interesting to explore combinations between our model and problem, and the classical problems of reliable communication over unreliable channels and networks. Furthermore, since our work requires a shared secret key, it may be desirable to merge it with protocols using shared secret key for confidentiality and authentication, such as SSL/TLS and IP-Sec.

\section{References}

[1] D. G. Andersen. Mayday: Distributed filtering for Internet services. In Proceedings of the 4th USENIX Symposium on Internet Technologies and Systems (USITS), 2003.

[2] K. Argyraki and D. R. Cheriton. Active Internet traffic filtering: Real-time response to denial-of-service attacks. In Proceedings of the USENIX Annual Technical Conference, April 2005. 
[3] R. Atkinson. Security architecture for the Internet Protocol. RFC 2401, IETF, 1998.

[4] G. Badishi, I. Keidar, and A. Sasson. Exposing and eliminating vulnerabilities to denial of service attacks in secure gossip-based multicast. In The International Conference on Dependable Systems and Networks (DSN), pages 223-232, June.July 2004.

[5] M. Collins and M. K. Reiter. An empirical analysis of target-resident DoS filters. In Proceedings of the 2004 IEEE Symposium on Security and Privacy, pages 103-114, May 2004.

[6] CSI/FBI. Computer crime and security survey, 2003.

[7] V. D. Gligor. Guaranteeing access in spite of service-flooding attacks. In the Security Protocols Workshop, 2003.

[8] O. Goldreich, S. Goldwasser, and S. Micali. How to construct random functions. Journal of the Association for Computing Machinery, 33(4):792-807, 1986.

[9] C. Jin, H. Wang, and K. G. Shin. Hop-count filtering: an effective defense against spoofed DDoS traffic. In V. Atluri and P. Liu, editors, Proceedings of the 10th ACM Conference on Computer and Communication Security (CCS-03), pages 30-41, New York, Oct. 27-30 2003. ACM Press.

[10] J. Jung, B. Krishnamurthy, and M. Rabinovich. Flash crowds and denial of service attacks: Characterization and implications for CDNs and web sites. In Proceedings of the International World Wide Web Conference, pages 252-262. IEEE, May 2002.

[11] Juniper Networks. The need for pervasive application-level attack protection.

[12] A. D. Keromytis, V. Misra, and D. Rubenstein. SOS: An architecture for mitigating DDoS attacks. Journal on Selected Areas in Communications, 21(1):176-188, 2004.

[13] B. Krishnamurthy and J. Wang. On network-aware clustering of Web clients. In Proceedings of the SIGCOMM, Aug. 2000.

[14] P. Mahajan, S. M. Bellovin, S. Floyd, J. Ioannidis, V. Paxson, and S. Shenker. Controlling high bandwidth aggregates in the network. Computer Communications Review, 32(3):62-73, July 2002. 
[15] D. Moore, G. Voelker, and S. Savage. Inferring Internet denial-of-service activity. In Proceedings of the 10th USENIX Security Symposium, pages 9-22, August 2001.

[16] NetContinuum. Web application firewall: How NetContinuum stops the 21 classes of web application threats.

[17] P-Cube. DoS protection.

[18] P-Cube. Minimizing the effects of DoS attacks.

[19] Riverhead Networks. Defeating DDoS attacks.

[20] S. M. Schwartz. Frequency hopping spread spectrum (fhss).

[21] A. Yaar, A. Perrig, and D. Song. Pi: A path identification mechanism to defend against DDoS attacks. In IEEE Symposium on Security and Privacy, May 2003.

[22] A. Yaar, A. Perrig, and D. Song. Siff: A stateless internet flow filter to mitigate ddos flooding attacks. In Proceedings of the IEEE Symposium on Security and Privacy, May 2004. 


\section{A Model and Specification of Port-Based Rationing Channel}

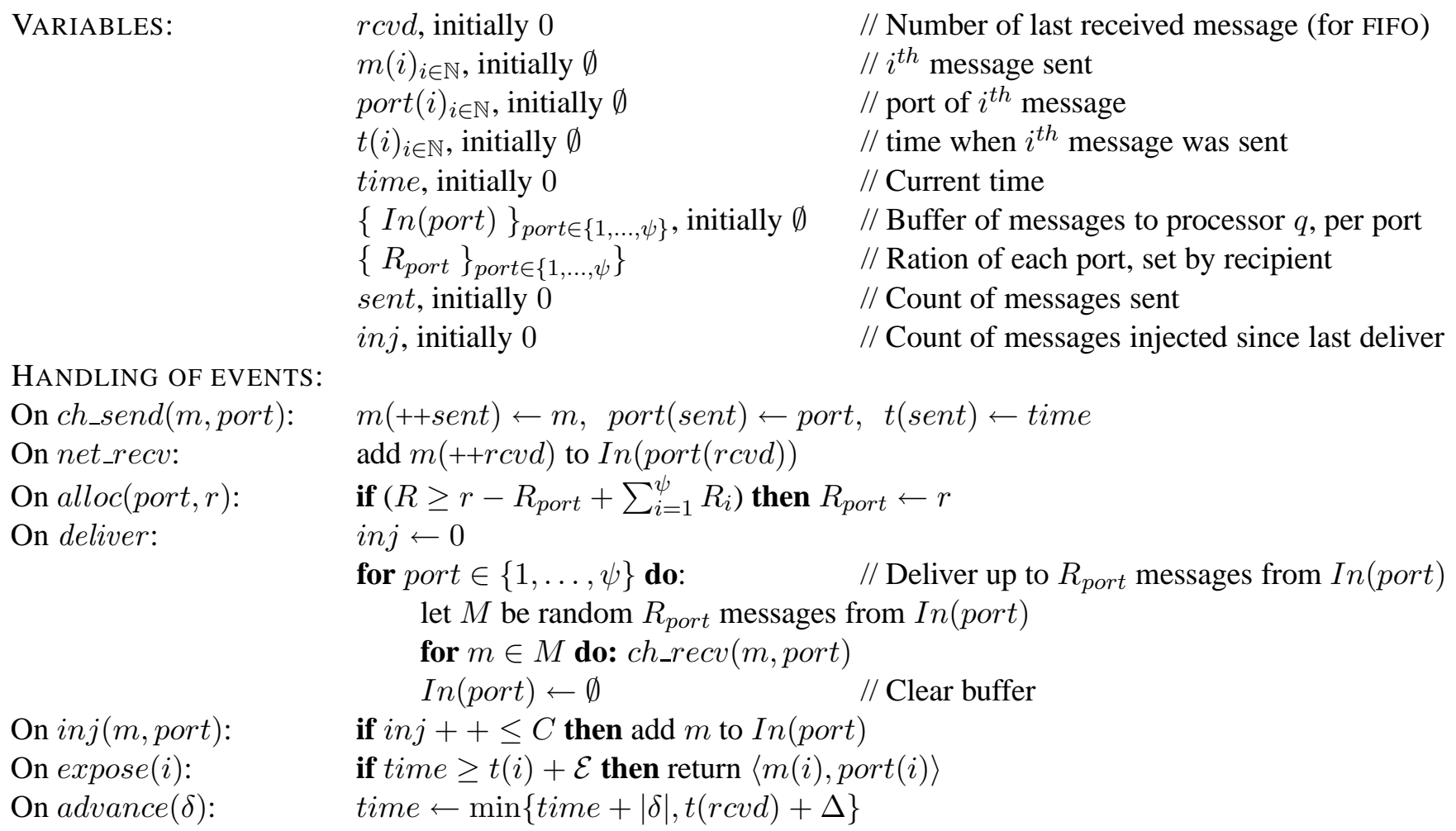

Figure 5: Port-based rationing channel for given $\Psi, R, C, \Phi, \Delta, \mathcal{E}$.

We model the system as a collection of interacting state machines. Each state machine is defined by its state (variables), set of possible initial states, and deterministic state transitions associated with input and output events. To allow machines to make random choices, initial states include random tapes.

We model the adversary as one of the deterministic state machines of which the system is composed. The adversary controls, among other things, the scheduling of events. That is, it defines the next event that will occur in any system state, as well as the progress of time (via the advance event). Thus, an execution of the system is completely defined by its initial state and number of steps ${ }^{4}$. The possible choices of random tapes define a probability space on executions.

A port-based rationing channel models a FIFO-ordered rate-limited communication channel with port-based message filtering. Figure 5 provides specifications for a channel from $A$ to $B$; we assume an equivalent channel is used from $B$ to $A$. The net recv event models the arrival of the next message from

\footnotetext{
${ }^{4}$ We encapsulate all non-determinism and randomness in the choice of random tapes.
} 
$A$ (in FIFO order) to the channel's buffer, allowing the adversary control of network latency (up to $\Delta$ ).

The recipient uses the alloc operation to designate ration values $R_{i}$ for ports $i \in\{1, \ldots, \psi\}$. If $R_{i}>0$ we say that port $i$ is open. We use $\operatorname{In}(i)$ to denote the set of messages in the input buffer designated with port $i$. The channel delivers all messages from $\operatorname{In}(i)$ if $|\operatorname{In}(i)| \leq R_{i}$, and a random subset of $R_{i}$ messages from $\operatorname{In}(i)$ if $|\operatorname{In}(i)|>R_{i}$.

The adversary can inject messages directly into the buffer using inj events, and can snoop on the contents of messages using expose events, under the restrictions above.

\section{B Channel Delivery Probability Analysis - Proofs of Lemmas}

We now prove the lemmas from Section 4. Since $a, C$, and $R$ are constants, denote $\mu_{B}\left(s_{k}\right)=\mu_{B}\left(a, C, R, s_{k}\right)$.

For brevity, denote $s_{k}=\left(c_{p_{1}}, c_{p_{2}}, \ldots, c_{p_{k}}\right)$ as the strategy where $p_{1}, p_{2}, \ldots, p_{k}$ are the ports that the attacker attacks at rates of $c_{p_{1}}, c_{p_{2}}, \ldots, c_{p_{k}}$ messages, resp., where $\sum_{i=1}^{k} c_{p_{i}}=C, c_{p_{i}}>0$. Assume that $\forall i c_{p_{i}} \geq R-a+1$ (otherwise, even if $p_{i}=p$, the probability of $B$ receiving $A$ 's messages is exactly 1 ).

Proposition 1. For fixed $k, a, C, R$, and $s_{k}$ as defined above, $\mu_{B}\left(a, C, R, s_{k}\right)=\frac{\psi-k}{\psi}+\frac{1}{\psi} \sum_{i=1}^{k} \frac{R}{c_{p_{i}}+a}$.

Proof. The probability that $B$ does not deliver $A$ 's message is: $\sum_{i=1}^{k} \operatorname{Pr}\left[p_{i}=p\right] \cdot\left(1-\frac{R}{c_{p_{i}}+a}\right)=$ $\frac{1}{\psi} \sum_{i=1}^{k}\left(1-\frac{R}{c_{p_{i}}+a}\right)=\frac{k}{\psi}-\frac{1}{\psi} \sum_{i=1}^{k} \frac{R}{c_{p_{i}}+a}$. Thus, the delivery probability is $\frac{\psi-k}{\psi}+\frac{1}{\psi} \sum_{i=1}^{k} \frac{R}{c_{p_{i}}+a}$.

Lemma 5. Under a blind attack with strategy $s_{k}=\left(c_{p_{1}}, c_{p_{2}}, \ldots, c_{p_{k}}\right)$ for fixed $k, a, C$, and $R$, the worst (i.e., minimal) expected delivery probability of the system is achieved when $\forall i c_{p_{i}}=\frac{C}{k}$.

Proof. By Proposition 1, $\mu_{B}\left(s_{k}\right)=\frac{\psi-k}{\psi}+\frac{1}{\psi} \sum_{i=1}^{k} \frac{R}{c_{p_{i}}+a}$. Calculating the partial derivatives of $\mu_{B}\left(s_{k}\right)$ we get that $\frac{\partial \mu_{B}\left(s_{k}\right)}{\partial c_{p_{i}}}=\frac{1}{\psi} \cdot \frac{-R}{\left(c_{p_{i}}+a\right)^{2}}$, i.e., $\mu_{B}\left(s_{k}\right)$ is monotonically decreasing as we increase $c_{p_{i}}$ and keep $c_{p_{j}}$ the same for $j \neq i$. Thus, the attacker wants to increase $c_{p_{i}}$ to decrease the delivery probability of the communication channel. However, we have the constraint $\sum_{i=1}^{k} c_{p_{i}}=C$. Integrating this constraint into our delivery probability function using a Lagrange coefficient denoted by $\beta$ gives:

$$
\mu_{B^{\prime}}\left(s_{k}\right)=\frac{\psi-k}{\psi}+\frac{1}{\psi} \sum_{i=1}^{k} \frac{R}{c_{p_{i}}+a}+\beta\left(\sum_{i=1}^{k} c_{p_{i}}-C\right)
$$


We now look for an extremum point by comparing the partial derivatives of $\mu_{B^{\prime}}\left(s_{k}\right)$ to zero:

$$
\begin{aligned}
\frac{\partial \mu_{B^{\prime}}\left(s_{k}\right)}{\partial c_{p_{i}}} & =0 \\
\frac{1}{\psi} \cdot \frac{-R}{\left(c_{p_{i}}+a\right)^{2}}+\beta & =0 \\
\beta & =\frac{R}{\psi\left(c_{p_{i}}+a\right)^{2}} \\
\left(c_{p_{i}}+a\right)^{2} & =\frac{R}{\psi \beta} \\
c_{p_{i}} & =\sqrt{\frac{R}{\psi \beta}}-a
\end{aligned}
$$

Putting the values of $c_{p_{i}}$ into the constraint equation $C=\sum_{i=1}^{k} c_{p_{i}}$ gives:

$$
\begin{aligned}
C & =\sum_{i=1}^{k}\left(\sqrt{\frac{R}{\psi \beta}}-a\right) \\
C & =k\left(\sqrt{\frac{R}{\psi \beta}}-a\right) \\
\left(\frac{C}{k}+a\right)^{2} & =\frac{R}{\psi \beta} \\
\beta & =\frac{R}{\psi\left(\frac{C}{k}+a\right)^{2}}
\end{aligned}
$$

Going back to the equation for $c_{p_{i}}$ we get:

$$
c_{p_{i}}=\sqrt{\frac{R}{\psi \cdot \frac{R}{\psi\left(\frac{C}{k}+a\right)^{2}}}}-a=\sqrt{\left(\frac{C}{k}+a\right)^{2}}-a=\frac{C}{k}
$$

This result also fits our constraint $c_{p_{i}}>0$, and we have an extremum point for $\mu_{B}\left(s_{k}\right)$ at $c_{p_{i}}=\frac{C}{k}$. (We note that $\frac{C}{k}$ might not be an integer, but since we want a lower bound, this does not make a difference.) We denote this extremum point by $s_{k}^{*}$. Now we need to show that $s_{k}^{*}$ is a minimum point. If we show that $\mu_{B}\left(s_{k}\right)$ is convex, then from Kuhn-Tucker Theorem we get that $s_{k}^{*}$ is a global minimum point. We proceed by showing that $\mu_{B}\left(s_{k}\right)$ is convex. 
We have already shown that $\frac{\partial \mu_{B}\left(s_{k}\right)}{\partial c_{p_{i}}}=\frac{R}{\psi} \cdot \frac{-1}{\left(c_{p_{i}}+a\right)^{2}}$. We get that $\mu_{B}\left(s_{k}\right)$ is twice continuously differentiable, and the second derivative is:

$$
\frac{\partial^{2} \mu_{B}\left(s_{k}\right)}{\partial c_{p_{i}} \partial c_{p_{j}}}= \begin{cases}0 & i \neq j \\ \frac{R}{\psi} \cdot \frac{2\left(c_{p_{i}}+a\right)}{\left(c_{p_{i}}+a\right)^{4}} & i=j\end{cases}
$$

We get that the Hessian of $\mu_{B}\left(s_{k}\right)$ is a positive diagonal matrix. Thus, $\mu_{B}\left(s_{k}\right)$ is convex, and from Kuhn-Tucker Theorem, $\mu_{B}\left(s_{k}^{*}\right)$ is a global minimum of the delivery probability function $\mu_{B}\left(s_{k}\right)$.

From Proposition 1 and Lemma 5 we get:

Corollary 2. Under a blind attack, if $k, a, C$, and $R$ are fixed, then the expected delivery probability for $s_{k} \in S_{k}$ is bounded from below as follows: $\mu_{B}\left(a, C, R, s_{k}\right) \geq \frac{\psi-k}{\psi}+\frac{1}{\psi} \cdot \sum_{i=1}^{k} \frac{R}{\frac{C}{k}+a}=\frac{\psi-k}{\psi}+\frac{1}{\psi} \cdot \frac{k R}{\frac{C}{k}+a}=$ $\frac{\psi-k}{\psi}+\frac{k^{2} R}{\psi(C+k a)}$.

We now define $\mu_{B}(k) \triangleq \min _{s_{k} \in S_{k}} \mu_{B}\left(s_{k}\right)$. We get that for each $k$ :

$$
\mu_{B}(k)=\frac{\psi-k}{\psi}+\frac{R}{\psi} \cdot \frac{k^{2}}{C+k a}
$$

To find a lower bound, we continue this analysis as if $k$ is continuous. The derivative of $\mu_{B}(k)$ is then:

$\mu_{B}^{\prime}(k)=\frac{-1}{\psi}+\frac{R}{\psi} \cdot \frac{2 k(C+k a)-k^{2} a}{(C+k a)^{2}}=\frac{R}{\psi} \cdot \frac{2 k C+k^{2} a}{(C+k a)^{2}}-\frac{1}{\psi}=\frac{R-1}{\psi}-\frac{R}{\psi} \cdot \frac{C^{2}+\left(2 k C+k^{2} a\right)(a-1)}{(C+k a)^{2}}$

We now prove two lemmas that show that $\mu_{B}(a, C, R)$ is bounded from below by the function $f(a, C, R)$ presented in Equation 1 .

Lemma 6. Let $R=a$, then an adversary with $C \geq \psi$ cannot decrease the expected delivery probability lower than $\frac{\psi a}{C+\psi a}$, and an adversary with $C \leq \psi$ cannot decrease the expected delivery probability lower than $1-\frac{C}{\psi(1+a)}$.

Proof. Let $R=a$. We get that $\mu_{B}^{\prime}(k)=\frac{R-1}{\psi}-\frac{R}{\psi} \cdot \frac{C^{2}+\left(2 k C+k^{2} R\right)(R-1)}{(C+k R)^{2}}$. We now show that $\mu_{B}^{\prime}(k)<0$ :

$$
\frac{R-1}{\psi}-\frac{R}{\psi} \cdot \frac{C^{2}+\left(2 k C+k^{2} R\right)(R-1)}{(C+k R)^{2}} \stackrel{?}{<} 0
$$




$$
\begin{aligned}
(C+k R)^{2}(R-1) & \stackrel{?}{\leq} R C^{2}+R(R-1)\left(2 k C+k^{2} R\right) \\
\left(C^{2}+2 k R C+k^{2} R^{2}\right)(R-1) & \stackrel{?}{\leq} R C^{2}+(R-1)\left(2 k C R+k^{2} R^{2}\right) \\
C^{2}(R-1) & \stackrel{?}{\leq} R C^{2} \\
0 & \stackrel{?}{\leq} C^{2}
\end{aligned}
$$

Clearly, the last inequality holds, and we get that $\mu_{B}(k)$ monotonically decreases as $k$ increases. Thus, the adversary wants to choose $k$ as large as possible. Ideally, $k=\psi, C \geq \psi(R-a+1)=\psi$ and we get:

$$
\mu_{B}(a, R, C) \geq \frac{a}{\psi} \cdot \frac{\psi^{2}}{C+\psi a}=\frac{\psi a}{C+\psi a}
$$

However, this attack requires substantial strength from the adversary, i.e., the adversary needs to be more than $\psi$ times stronger than $B$. If $C \leq \psi(R-a+1)=\psi$ we get that $k=\frac{C}{R-a+1}=C$. The resulting degraded delivery probability is:

$\mu_{B}(a, R, C) \geq \frac{\psi-C}{\psi}+\frac{a}{\psi} \cdot \frac{C^{2}}{C(1+a)}=\frac{\psi(1+a)-C(1+a)+a C}{\psi(1+a)}=1-\frac{C}{\psi(1+a)} \geq 1-\frac{\psi}{\psi(1+a)}=1-\frac{1}{1+a}$

Lemma 7. Let $a<R$. Then an adversary with $C \geq \frac{\psi a}{\sqrt{\frac{R}{R-a}}-1}$ cannot decrease the expected delivery probability lower than $\frac{\psi R}{C+\psi a}$, and an adversary with $C \leq \frac{\psi a}{\sqrt{\frac{R}{R-a}}-1}$ cannot decrease the expected delivery probability lower than $\frac{\psi a-C\left(\sqrt{\frac{R}{R-a}}-1\right)}{\psi a}+\frac{R}{\psi} \cdot \frac{C\left(\sqrt{\frac{R}{R-a}}-1\right)^{2}}{a^{2} \sqrt{\frac{R}{R-a}}}$.

Proof. Since $a<R$, we get $R \geq 2$. Let us find the value of $k$ that minimizes the delivery probability:

$$
\begin{aligned}
\mu_{B}^{\prime}(k) & =0 \\
\frac{R-1}{\psi}-\frac{R}{\psi} \cdot \frac{C^{2}+\left(2 k C+k^{2} a\right)(a-1)}{(C+k a)^{2}} & =0 \\
(C+k a)^{2}(R-1)-R C^{2}-R\left(2 k C+k^{2} a\right)(a-1) & =0 \\
2 k C R+k^{2} a R-2 k C a-k^{2} a^{2}-C^{2} & =0
\end{aligned}
$$




$$
\begin{aligned}
2 k C(R-a)+k^{2} a(R-a)-C^{2} & =0 \\
a k^{2}+2 C k-\frac{C^{2}}{R-a} & =0
\end{aligned}
$$

Since $k>0$, we get that the solution is:

$$
k=\frac{-2 C+\sqrt{4 C^{2}+\frac{4 C^{2} a}{R-a}}}{2 a}=\frac{-2 C+\sqrt{\frac{4 C^{2} R}{R-a}}}{2 a}=\frac{C\left(\sqrt{\frac{R}{R-a}}-1\right)}{a}
$$

Obviously, this value of $k$ is not an integer. However, we use it to bound the minimum delivery probability under a blind DoS attack. First, we need to show that this value of $k$ is indeed a minimum point. We do this by showing that the second derivative of $\mu_{B}(k)$ is always positive:

$$
\mu_{B}^{\prime \prime}(k)=\frac{R}{\psi} \cdot \frac{2 x(C+k x)\left[C^{2}+\left(2 k C+k^{2} a\right)(a-1)\right]-(2 C+2 k a)(a-1)}{(C+k)^{4}}
$$

It suffices to show that the numerator is always positive. I.e., we need to show:

$$
a(2 C+2 k a)\left[C^{2}+\left(2 k C+k^{2} a\right)(a-1)\right]>(2 C+2 k a)(a-1)
$$

This is clearly true, since $a \geq 1, k \geq 1, C \geq 1$, and we get $a\left[C^{2}+\left(2 k C+k^{2} a\right)(a-1)\right]>a-1$. Thus, $\mu_{B}^{\prime \prime}(k)$ is always positive, and we have found a minimum point.

We also need the found $k$ to be in range. Clearly, $k>0$. We still need to show that $k \leq \frac{C}{R-a+1}$ :

$$
\begin{aligned}
k & \stackrel{?}{\leq} \frac{C}{R-a+1} \\
\frac{C\left(\sqrt{\frac{R}{R-a}}-1\right)}{a} & \stackrel{?}{\leq} \frac{C}{R-a+1} \\
\sqrt{\frac{R}{R-a}} & \stackrel{?}{\leq} \frac{a}{R-a+1}+1 \\
\frac{R}{R-a} & \stackrel{?}{\leq} \frac{(R+1)^{2}}{(R-a+1)^{2}} \\
R(R+1)^{2}-2 R a(R+1)+R a^{2} & \stackrel{?}{\leq} R(R+1)^{2}-a(R+1)^{2}
\end{aligned}
$$




$$
\begin{aligned}
R a-2 R^{2} & \stackrel{?}{\leq}-R^{2}-1 \\
a & \stackrel{?}{\leq} R-\frac{1}{R}
\end{aligned}
$$

The last inequality holds since $a<R, a$ is an integer, and $R \geq 2$. Thus, $k \leq \frac{C}{R-a+1}$.

We can now bound the expected delivery probability $\mu(a, R, C)$ from below. For the case where $k=\frac{C\left(\sqrt{\frac{R}{R-a}}-1\right)}{a} \leq \psi$ we get:

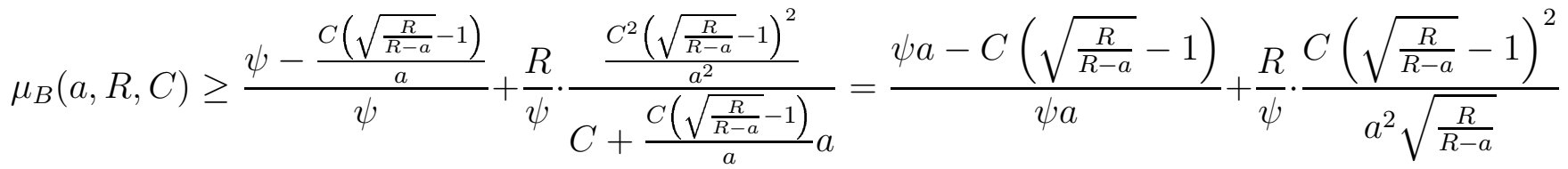

For the case where $\frac{C\left(\sqrt{\frac{R}{R-a}}-1\right)}{a}>\psi$, since $\mu_{B}(k)$ has just one extremum point, and it is a minimum point with $k>\psi$, we get that the attacker's best strategy is to choose $k=\psi$, and we get:

$$
\mu_{B}(a, R, C) \geq \frac{\psi-\psi}{\psi}+\frac{\psi^{2} R}{\psi(C+\psi a)}=\frac{\psi R}{C+\psi a}
$$

Note that we got the same result for $R=a$ and $k=\psi$. However, the conditions for choosing $k=\psi$ are different. For $R=a$ we choose $k=w$ if $C \geq w$. For $R>a$ we choose $k=\psi$ if $\frac{C\left(\sqrt{\frac{R}{R-a}}-1\right)}{a}>\psi$.

We conclude the following lemma:

Lemma 3. $\mu_{B}(a, C, R)$ is bounded from below by the following function $f(a, C, R)$ :

$$
f(a, C, R)= \begin{cases}\frac{\psi a}{C+\psi a} & \text { if } R=a \text { and } C \geq \psi \\ 1-\frac{C}{\psi(1+a)} & \text { if } R=a \text { and } C<\psi \\ \frac{\psi R}{C+\psi a} & \text { if } R>a \text { and } C \geq \frac{\psi a}{\sqrt{\frac{R}{R-a}}-1} \\ \frac{\psi a-C\left(\sqrt{\frac{R}{R-a}}-1\right)}{\psi a}+\frac{R}{\psi} \cdot \frac{C\left(\sqrt{\frac{R}{R-a}}-1\right)^{2}}{a^{2} \sqrt{\frac{R}{R-a}}} & \text { if } R>a \text { and } C<\frac{\psi a}{\sqrt{\frac{R}{R-a}}-1} \\ 0 & \text { otherwise }\end{cases}
$$




\section{Ack-Based Protocol - Proof of Correctness}

Invariant 1. Let $P_{A}$ and $P_{B}$ be the $P$ counters that $A$ and $B$ hold in the ack-based protocol, respectively. The probability that $P_{B}-P_{A} \in\{0,1\}$ is 1 up to a polynomially-negligible factor.

Proof. After the initialization stage $P_{A}=P_{B}$, and the property $P_{B}-P_{A} \in\{0,1\}$ holds.

When the counters are equal, the part of the protocol that may update them proceeds as follows:

1. $A$ sends a message to $B$ on port $P R F_{S_{A B}}\left(P_{A} \mid\right.$ "data").

2. If the message reaches $B$ in a valid state, $B$ adds 1 to $P_{B}$ and sends an acknowledgment back to $A$ on port $P R F_{S_{B A}}\left(P_{B} \mid\right.$ "ack").

3. If the ack reaches $A$ in a valid state, $A$ adds 1 to $P_{A}$.

If steps 2 and 3 complete successfully, both counters advance by 1 and remain equal to each other. If step 2 fails (message dropped or modified in transit), both counters remain unchanged. If step 2 succeeds but step 3 fails (ack lost or changed in transit), $P_{B}$ is incremented by 1 , but $P_{A}$ remains the same. Thus, if $P_{A}=P_{B}$, the next change of counters will still maintain the property $P_{B}-P_{A} \in\{0,1\}$.

Now suppose we have reached the state where $P_{B}=P_{A}+1$. The portion of the protocol that may update the counters proceeds as follows:

1. $A$ sends a message to $B$ on port $P R F_{S_{A B}}\left(P_{A} \mid\right.$ "data").

2. If the message reaches $B$ in a valid state, $B$ sends an ack back to $A$ on port $P R F_{S_{B A}}\left(P_{B}-1 \mid\right.$ "ack").

3. If the ack reaches $A$ in a valid state, $A$ adds 1 to $P_{A}$.

If steps 2 and 3 complete successfully, $P_{A}$ advances by 1 and the counters become equal to each other. If steps 2 or 3 fail (messages dropped or are not valid), both counters remain unchanged. Thus, if $P_{B}=P_{A}+1$, the next change of counters will still maintain the property $P_{B}-P_{A} \in\{0,1\}$.

The only way to break this invariant is if the attacker makes just one party advance its counter. This means that the adversary has to fabricate a message so one party will think it is valid. Thus, the attacker 
needs to guess both the port number and the authentication information attached to each message. The probability that the attacker succeeds in doing so is a polynomially-negligible factor.

Theorem 1. When using the ack-based protocol, the probability that a data message that A sends to port p arrives when $p$ is open is 1 up to a polynomially-negligible factor.

Proof. According to Invariant 1, when $A$ sends a data message to $B$, either $P_{A}=P_{B}$ or $P_{B}=P_{A}+1$, with probability 1 up to a polynomially-negligible factor.

For the first case, let $M$ be a message $A$ sends to $B$ when $P_{A}=P_{B}$. Since $B$ always opens two ports for data, we need to show that $P_{B}$ does not increase by more than one until $M$ actually reaches $B$. Since the link maintains the FIFO semantics, messages sent after $M$ was sent cannot change the value of $P_{B}$ before $M$ reaches $B$. The only messages that can change $P_{B}$ are messages that preceded $M$ but reached $B$ only after $M$ was sent.

According to the protocol, $P_{B}$ increases by one iff $B$ receives a data message from $A$ that was sent using the counter $P_{A}=P_{B}$. Furthermore, all messages preceding $M$ were sent using a counter that is less than or equal to $P_{A}$. It follows that $P_{B}$ can only increase by one from the time $M$ leaves $A$ until it reaches $B$.

Consider now the second case where $M$ was sent when $P_{B}=P_{A}+1$. Since $B$ only opens two ports for data, we need to show that $P_{B}$ does not change at all. Again, since the link has FIFO semantics, $P_{B}$ can only change by messages preceding $M$ that reach $B$ after $M$ was sent but before it reaches $B$. However, such messages have counters that are less than or equal to $P_{A}$, and thus strictly less than $P_{B}$. According to the protocol, messages sent with such counters do not affect the value of $P_{B}$.

\section{Ack-Based with Reinitializations - Proof of Correctness}

Theorem 2. When using the ack-based protocol with proactive reinitializations, the probability that a data message that A sends to port p arrives when $p$ is open is 1 up to a polynomially-negligible factor.

Proof. From Theorem 1 we get that if $A$ and $B$ both use the ack-based protocol initialized with seed, 
then messages sent by $A$ arrive to open ports at $B$. To complete the proof, we need to show the following:

1. When $A$ reinitializes the protocol with a new seed, $B$ has already started running an ack-based protocol instance using the same seed.

2. $B$ does not terminate a protocol instance while it may still receive messages corresponding to that instance.

For the first property, let us look at some real time $t_{n}^{A}$ when $A$ reinitializes the protocol, where $t_{A}\left(t_{n}^{A}\right)=n \delta, n \in \mathbb{N}$. From the bounded drift assumption we get the bound $t_{n}^{A} \geq n \delta-\Phi$. The seed corresponding to the initialization at $t_{n}^{A}$ is $\frac{t_{A}\left(t_{n}^{A}\right)}{\delta}=n$. Now let us look at the real time $t_{n}^{B}$ in which $B$ starts a new ack-based protocol instance with the seed $n$. This happens when $t_{B}\left(t_{n}^{B}\right)+2 \Phi=n \delta$, i.e., when $t_{B}\left(t_{n}^{B}\right)=n \delta-2 \Phi$. Using the bounded drift assumption we get the bound $t_{n}^{B} \leq n \delta-2 \Phi+\Phi=$ $n \delta-\Phi \leq t_{n}^{A}$.

For the second property, let us look at seed $n$ again. $A$ terminates the instance with seed $n$ at real time $t_{n+1}^{A}$. The last packet sent using the ack-based protocol initialized with seed $n$ inevitably reaches $B$ before real time $t_{n+1}^{A}+\Delta . B$ terminates the ack-based protocol instance in either one of the following two cases:

1. At time $t_{B}\left(t_{n+1}^{B}\right)+4 \Phi+\Delta$.

2. $\Delta$ time units after receiving the first message for a newer ack-based protocol instance.

For the first case, we get $t_{n+1}^{B} \geq(n+1) \delta-2 \Phi-\Phi+4 \Phi+\Delta=(n+1) \delta+\Phi+\Delta \geq t_{n+1}^{A}+\Delta$. For the second case, we observe that if a message for a newer instance of the ack-based protocol has arrived, then $A$ is no longer sending messages with instances initialized with older seeds. However, the varying message propagation delay means that messages from older protocol instances can take up to $\Delta$ time units to arrive, while the new message might have taken negligible time to arrive. 